\title{
Studies on Fractional Differential Operators of Two Parameters in a Complex Domain
}

\author{
Rabha W. Ibrahim ${ }^{1, *}$, Hamid A. Jalab ${ }^{2}$ \\ ${ }^{1}$ Institute of Mathematical Sciences, University Malaya, 50603, Malaysia \\ ${ }^{2}$ Faculty of Computer Science and Information Technology, University, Malaya, 50603, Malaysia \\ *Corresponding author: rabhaibrahim@yahoo.com \\ Received December 18, 2015; Revised February 01, 2016; Accepted February 09, 2016
}

\begin{abstract}
This study deals with a generalization for fractional differential operators in a complex domain based on the extended Beta function. Stipulations are imposed for these generalized operators such as the upper bounds. Other possessions for the above operator are also prepared. In addition, implementations of these operators are introduced and suggested in the geometric function theory (GFT). Sufficient conditions are imposed for functions to be univalent.
\end{abstract}

Keywords: Fractional calculus, fractional differential operator, fractional differential equation, analytic function

Cite This Article: Rabha W. Ibrahim, and Hamid A. Jalab, "Studies on Fractional Differential Operators of Two Parameters in a Complex Domain." Turkish Journal of Analysis and Number Theory, vol. 4, no. 1 (2016): 1-7. doi: 10.12691/tjant-4-1-1.

\section{Introduction}

Newly, the subject of fractional calculus has established consideration and presentations in all mathematical branches (pure and applied). One of these significant concepts is the theory of analytic functions. The conventional descriptions of fractional operators and their generalizations have successfully been realized in finding, for example, the coefficient estimates [1], the classification properties, distortion inequalities [2] and convolution constructions for numerous subclasses of analytic functions.

In [3], Srivastava and Owa, contributed designations for fractional operators (integral and derivative) in the complex z-plane $\mathbb{C}$ as follows: the fractional derivative of order $\wp$ is defined, for a function $\phi(z)$ by

$$
D_{z}^{\wp} \phi(z):=\frac{1}{\Gamma(1-\wp)} \frac{d}{d z} \int_{0}^{z} \frac{\phi(\zeta)}{(z-\zeta)} d \zeta ; 0 \leq \wp<1,
$$

where the function $\phi(z)$ is analytic in simply-connected region of the complex z-plane $\mathbb{C}$ containing the origin and the multiplicity of $(z-\zeta)^{-\wp}$ is removed by requiring $\log (z-\zeta)$ to be real when $(z-\zeta)>0$. While the fractional integral of order $\}$ is defined, for a function $\phi(z)$, by

$$
I_{z}^{\wp} \phi(z):=\frac{1}{\Gamma(\wp)} \int_{0}^{z} \phi(\zeta)(z-\zeta)^{\wp-1} d \zeta ; \wp>0,
$$

where the function $\phi(z)$ is analytic in simply-connected region of the complex z-plane $(\mathbb{C})$ containing the origin and the multiplicity of $(z-\zeta)^{\wp-1}$ is removed by requiring $\log (z-\zeta)$ to be real when $(z-\zeta)>0$.

Ibrahim extended (1) and (2) to involve two fractional parameters as follows [4]:

$$
\begin{aligned}
D_{Z}^{\wp, \varsigma} \phi(z) & :=\frac{(\varsigma+1)^{\wp}}{\Gamma(1-\wp)} \frac{d}{d z} \int_{0}^{z} \frac{\zeta^{\varsigma} \phi(\zeta)}{\left(z^{\varsigma+1}-\zeta^{\varsigma+1}\right)^{\wp}} d \zeta ; \\
0 & \leq \wp<1, \varsigma>-1
\end{aligned}
$$

and

$$
\begin{aligned}
I_{Z}^{\wp, \varsigma} \phi(z) & :=\frac{(\varsigma+1)^{1-\wp}}{\Gamma(\wp)} \frac{d}{d z} \int_{0}^{z}\left(\begin{array}{l}
z^{\varsigma+1} \\
-\zeta^{\varsigma+1}
\end{array}\right)^{\wp-1} \zeta^{\varsigma} \phi(\zeta) d \zeta,(4) \\
\wp>0, \varsigma>-1 . &
\end{aligned}
$$

Recently, Srivastava et al. [5] extended the following Beta function:

$$
\begin{aligned}
& \mathbf{B}_{p}^{\alpha, \beta, \kappa, \mu}(a, b) \\
& =\int_{0}^{1} \tau^{\alpha-1}(1-\tau)^{b-1}{ }_{1} F_{1}\left(\alpha ; \beta ;-\frac{p}{\tau^{\kappa}(1-\tau)^{\mu}}\right) d \tau, \\
& \left(\begin{array}{l}
\kappa \geq 0, \mu \geq 0, \min \{\mathfrak{R}(\alpha), \mathfrak{R}(\beta)\}>0, \\
\Re(a)>-\Re(\kappa \alpha), \mathfrak{R}(b)>-\mathfrak{R}(\mu \beta)
\end{array}\right) .
\end{aligned}
$$

It is clear that when $p=0$ then (5) reduces to the familiar beta function $B(a, b)$.

Definition 1.1 The extended Srivastava-Owa fractional derivative of $\phi(z)$ of order $\wp$ is formulated by 


$$
\begin{aligned}
& D_{z, p, \alpha, \beta}^{\wp, \kappa, \mu} \phi(z) \\
& :=\frac{1}{\Gamma(1-\wp)} \frac{d}{d z} \int_{0}^{z} \frac{\phi(\zeta)}{(z-\zeta)^{\wp}}{ }_{1} F_{1}\left(\alpha ; \beta ;-\frac{p z^{\kappa+\mu}}{\zeta^{\kappa}(z-\zeta)^{\mu}}\right) d \zeta, \\
& \left(\begin{array}{l}
0 \leq \wp<1, \kappa \geq 0, \mu \geq 0, \min \{\Re(\alpha), \Re(\beta)\}>0, \\
\Re(a)>-\Re(\kappa \alpha), \Re(b)>-\Re(\kappa \beta)
\end{array}\right) .
\end{aligned}
$$

Obviously, when $p=0$, the operator (6) reduces to the operator (1).

Definition 1.2 The extended Srivastava-Owa-Ibrahim fractional derivative of $\phi(z)$ of order $\wp$ and $\varsigma$ is defined by

$$
\begin{aligned}
& D_{z, p, \alpha, \beta}^{\wp, \varsigma, \mu, \mu} \phi(z) \\
& :=\frac{(\wp+1)^{\wp}}{\Gamma(1-\wp)} \frac{d}{d z} \int_{0}^{z}\left[\begin{array}{c}
\left(z^{\varsigma+1}-\zeta^{\varsigma+1}\right)^{\wp} \\
{ }_{1} F_{1}\left(\alpha ; \beta ;-\frac{p z}{\zeta^{\kappa}\left(z^{\varsigma+1}-\zeta^{\varsigma+1}\right)^{\mu}}\right)
\end{array}\right] d \zeta, \\
& \left(\begin{array}{l}
(0 \leq \wp<1, \varsigma>-1, \kappa \geq 0, \mu \geq 0), \\
\min \{\Re(\alpha), \Re(\beta)\}>0, \\
\Re(a)>-\Re(\kappa \alpha), \Re(b)>-\Re(\kappa \beta)
\end{array}\right) .
\end{aligned}
$$

Obviously, when $p=0$, the operator (7) reduces to the operator (3).

Example 1.1 We find the generalized derivative of the function $\phi(z)=z^{v}, v \in \mathbb{Z}$. Assume that

$$
\eta:=\frac{\zeta}{z}
$$

thus, we obtain

$$
\begin{aligned}
& D_{z, p, \alpha, \beta}^{\wp, \kappa, \mu} z^{v} \\
& =\frac{1}{\Gamma(1-\wp)} \frac{d}{d z} \int_{0}^{z} \frac{\zeta^{v}}{(z-\zeta)^{\wp}}{ }_{1} F_{1}\left(\alpha ; \beta ;-\frac{p z^{\kappa+\mu}}{\zeta^{\kappa}(z-\zeta)^{\mu}}\right) d \zeta \\
& =\frac{1}{\Gamma(1-\wp)} \frac{d}{d z} z^{(1-\wp)+v} \int_{0}^{1}\left[{ }_{1} F_{1}\left(\alpha ; \beta ;-\frac{p}{\eta^{\kappa}(1-\eta)^{\mu}}\right)\right] d \eta \\
& =\frac{\Gamma(v+1)}{\Gamma(v+1-\wp)} \frac{\mathbf{B}_{p}^{\alpha, \beta, \kappa, \mu}(v+1,1-\wp)}{\mathbf{B}(v+1,1-\wp)} z^{\nu-\wp .} .
\end{aligned}
$$

When $p=0$, we obtain

$$
D_{z}^{\wp} z^{v}=\frac{\Gamma(v+1)}{\Gamma(v+1-\wp)} z^{v-\wp} .
$$

Example 1.2 We compute the generalized derivative of two parameters for the function $\phi(z)=z^{v}, v \in \mathbb{R}$. Suppose

$$
\chi:=\left(\frac{\zeta}{z}\right)^{\mu+1}
$$

then we get

$$
\begin{aligned}
& D_{z, p, \alpha, \beta}^{\wp, \varsigma, \kappa, \mu} z^{\nu} \\
& =\frac{(\wp+1)^{\wp}}{\Gamma(1-\wp)} \frac{d}{d z} \int_{0}^{z}\left[\begin{array}{c}
\frac{\zeta^{\varsigma} \zeta^{\mu}}{\left(z^{\varsigma+1}-\zeta^{\varsigma+1}\right)^{\wp}} \\
{ }_{1} F_{1}\left(\alpha ; \beta ;-\frac{p z}{\left.\zeta^{(\varsigma+1}\right)_{(\kappa+\mu)}\left(z^{\varsigma+1}-\zeta^{\varsigma+1}\right)^{\mu}}\right)
\end{array}\right] d \zeta \\
& =\frac{(\wp+1)^{\wp}}{\Gamma(1-\wp)} \frac{d}{d z} z^{(1-\wp)(\varsigma+1)+v} \\
& \times \int_{0}^{1}\left[\begin{array}{c}
\chi^{\frac{v+\varsigma+1}{\varsigma+1}-1}(1-\chi)^{(1-\wp)-1} \\
{ }_{1} F_{1}\left(\alpha ; \beta ;-\frac{p}{\chi^{\kappa}(1-\chi)^{\mu}}\right)
\end{array}\right] d \chi \\
& =\frac{(\varsigma+1)^{\wp-1} \Gamma\left(\frac{v}{\varsigma+1}+1\right)}{\Gamma\left(\frac{v}{\varsigma+1}+1-\wp\right)} \frac{\mathbf{B}_{p}^{\alpha, \beta, \kappa, \mu}\left(\frac{v+\varsigma+1}{\varsigma+1}, 1-\wp\right)}{\mathbf{B}\left(\frac{v+\varsigma+1}{\varsigma+1}, 1-\wp\right)} \\
& \times z^{(1-\wp)(\varsigma+1)+v-1} .
\end{aligned}
$$

When $p=0, \varsigma=0$, we obtain (8).

In this study stipulations are imposed for the operators (6) and (7). Other possessions for the above operators are also prepared. In addition, implementations are introduced and suggested in GFT.

\section{Main Outcomes}

This section deals withe some applications of the new fractional differential operators (6) and (7) in view of GFT.

\subsection{Upper Bounds}

Let $\mathcal{A}$ denote the class of functions $\phi(z)$ normalized by

$$
\phi(z)=z+\sum_{n=2}^{\infty} \varphi_{2} z^{n}, \quad z \in U .
$$

A convolution product for two function $\phi, \psi \in \mathcal{A}$ is defined by

$$
\left(\phi^{*} \psi\right)(z)=z+\sum_{n=2}^{\infty} \varphi_{n} \omega_{n} z^{n}, \quad z \in U,
$$

where $\psi(z)=z+\sum_{n=2}^{\infty} \omega_{n} z^{n}$.

In addition, let $\mathcal{S}$ and $\mathcal{K}$ refer to the subclasses of $\mathcal{A}$ involving functions which are, respectively, univalent and convex in $U$. It is well known that; if the function $\phi(z)$ given by (9) is in the class $\mathcal{S}$, then

$$
\left|\varphi_{n}\right| \leq n, \quad n \in \mathbb{N} \backslash\{1\} .
$$

Equality holds for the Koebe function 


$$
\phi(z)=\frac{z}{(1-z)^{2}}, z \in U
$$

In addition, if the function $\phi(z)$ presented by (9) is in the class $\mathcal{K}$, then

$$
\varphi_{n} \leq 1, n \in \mathbb{N} .
$$

Equality holds for the function

$$
\phi(z)=\frac{z}{1-z}, \quad z \in U .
$$

In view of Examples 1.1 and 1.2, we have

$$
D_{z, p, \alpha, \beta}^{\wp, \kappa, \mu} \phi(z)=\sum_{n=1}^{\infty} a_{n} D_{z, p, \alpha, \beta}^{\wp, \kappa, \mu} z^{n}
$$

and

$$
D_{z, p, \alpha, \beta}^{\wp, \varsigma, \kappa, \mu} \phi(z)=\sum_{n=1}^{\infty} \varphi_{n} D_{z, p, \alpha, \beta}^{\wp, \varsigma, \kappa, \mu} z^{n}
$$

We need the following generalized hypergeometric functions in the sequel:

Definition 2.1 Based on the generalized Beta function, the extended Gauss hypergeometric function is formulated by [5]

$F_{p}^{\alpha, \beta, \kappa, \mu}(a, b ; c ; z)=\sum_{n=0}^{\infty}(a)_{n} \frac{\mathbf{B}_{p}^{\alpha, \beta, \kappa, \mu}(b+n, c-b)}{\mathbf{B}(b, c-b)} \frac{z^{n}}{n !}$,

$(z \in U, \min \mathfrak{R}(\alpha, \beta, \kappa, \mu)>0, \mathfrak{R}(c)>\mathfrak{R}(b), p \geq 0)$,

where $(x)_{n}=\frac{\Gamma(x+n)}{\Gamma(x)}, \quad(x)_{0}=1$, is the Pochhammer symbol. A further extension can be realized by the relation [6]

$$
\begin{aligned}
& F_{p}^{\alpha, \beta, \kappa, \mu}(a, b ; c ; z ; h) \\
& =\sum_{n=0}^{\infty} \frac{(a)_{n}(b)_{n}}{(c)_{n}} \frac{\mathbf{B}_{p}^{\alpha, \beta, \kappa, \mu}(b+n, c-b+h)}{\mathbf{B}(b+n, c-b+h)} \frac{z^{n}}{n !}, \\
& (z \in U, \min \mathfrak{R}(\alpha, \beta, \kappa, \mu)>0, \Re(c)>\Re(h)>h, p \geq 0) .
\end{aligned}
$$

Definition 2.2 The Fox-Wright generalization ${ }_{q} \Psi_{p}[z]$ of the hypergeometric ${ }_{q} F_{p}$ function defined by [7]

$$
\begin{aligned}
& { }_{q} \Psi_{p}\left[\begin{array}{l}
\left(a_{1}, \Lambda_{1}\right), \ldots,\left(a_{q}, \Lambda_{q}\right) \\
\left(b_{1}, \Xi_{1}\right), \ldots,\left(b_{p}, \Xi_{p}\right)
\end{array}\right] z \\
& ={ }_{q} \Psi_{p}\left[\left(a_{j}, \Lambda_{j}\right)_{1, q} ;\left(b_{j}, \Xi_{j}\right)_{1, p} ; z\right] \\
& :=\sum_{n=0}^{\infty} \frac{\Gamma\left(a_{1}+n \Lambda_{1}\right) \ldots \Gamma\left(a_{q}+n \Lambda_{q}\right)}{\Gamma\left(b_{1}+n \Lambda_{1}\right) \ldots \Gamma\left(b_{q}+n \Lambda_{q}\right)} \frac{z^{n}}{n !}
\end{aligned}
$$

where $\Lambda_{j}>0$ for all $j=1, \ldots, q, \Xi_{j}>0$ for all $j=1, \ldots, p$ and $1+\sum_{j=1}^{p} \Xi_{j}-\sum_{j=1}^{q} \Lambda_{j} \geq 0$ for suitable values $|z|<1$.

Theorem 2.1 Let $\phi \in \mathcal{S}$. Then

$$
\begin{gathered}
\left|D_{z, p, \alpha, \beta}^{\wp, \kappa, \mu} \phi(z)\right| \leq \frac{r^{1-\wp}}{\Gamma(2-\wp)} F_{p}^{\alpha, \beta, \kappa, \mu}(2,2 ; 2-\wp ; z ; m) \\
(r=|z| ; \quad z \in U ; m-1 \leq \wp<m, m \in \mathbb{N})
\end{gathered}
$$

where the equality holds true for the Koebe function.

Proof. Presume that the function $\phi(z) \in \mathcal{S}$ is given by (9). Then, by employing Example 1.1, we have

$$
\begin{aligned}
& D_{z, p, \alpha, \beta}^{\wp, \kappa, \mu} \phi(z)=\sum_{n=1}^{\infty} \varphi_{n} D_{z, p, \alpha, \beta}^{\wp, \kappa, \mu} z^{n}, \quad \varphi_{1}=1 \\
& =\sum_{n=1}^{\infty} \varphi_{n} \frac{\Gamma(n+1)}{\Gamma(n+1-\wp)} \frac{\mathbf{B}_{p}^{\alpha, \beta, \kappa, \mu}(n+1, m-\wp)}{\mathbf{B}(n+1, m-\wp)} z^{n-\wp} .
\end{aligned}
$$

Thus by using the fact $j^{\prime} n j \cdot n$; we obtain

$$
\begin{aligned}
& \left|D_{z, p, \alpha, \beta}^{\wp, \kappa, \mu} \phi(z)\right| \\
& \leq \sum_{n=1}^{\infty} \varphi_{n} \frac{\Gamma(n+1)}{\Gamma(n+1-\wp)} \frac{\mathbf{B}_{p}^{\alpha, \beta, \kappa, \mu}(n+1, m-\wp)}{\mathbf{B}(n+1, m-\wp)}|z|^{n-\wp} \\
& \leq r^{-\wp} \sum_{n=1}^{\infty} n \frac{\Gamma(n+1)}{\Gamma(n+1-\wp)} \frac{\mathbf{B}_{p}^{\alpha, \beta, \kappa, \mu}(n+1, m-\wp)}{\mathbf{B}(n+1, m-\wp)} r^{n} \\
& =r^{1-\wp} \sum_{n=0}^{\infty}(n+1) \frac{\Gamma(n+2)}{\Gamma(n+2-\wp)} \frac{\mathbf{B}_{p}^{\alpha, \beta, \kappa, \mu}(n+2, m-\wp)}{\mathbf{B}(n+2, m-\wp)} r^{n} \\
& =\frac{r^{1-\wp}}{\Gamma(2-\wp)} \sum_{n=0}^{\infty} \frac{(2)_{n}(2)_{n}}{(2-\wp)_{n}} \frac{\mathbf{B}_{p}^{\alpha, \beta, \kappa, \mu}(n+2, m-\wp)}{\mathbf{B}(n+2, m-\wp)} \frac{r^{n}}{n !} \\
& =\frac{r^{1-\wp}}{\Gamma(2-\wp)} F_{p}^{\alpha, \beta, \kappa, \mu}(2,2 ; 2-\wp ; r ; m) .
\end{aligned}
$$

This completes the proof.

In the same manner of Theorem 2.1, we have a distortion inequality involving the extended Gauss hypergeometric function, which is read by

Theorem 2.2 Let $\phi \in \mathcal{K}$. Then

$$
\begin{gathered}
\left|D_{z, \rho, \alpha, \beta}^{\wp, \mu, \mu} \phi(z)\right| \leq \frac{r^{1-\wp}}{\Gamma(2-\wp)} F_{p}^{\alpha, \beta, \kappa, \mu}(1,2 ; 2-\wp ; z ; 1) . \\
(r=|z| ; \quad z \in U ; 0<\wp<1),
\end{gathered}
$$

where the equality holds true for the Koebe function.

Proof. Suppose that the function $\phi(z) \in \mathcal{K}$, i.e. $\left|\varphi_{n}\right| \leq 1$ is written by (9). Then, we conclude

$$
\begin{aligned}
& \left|D_{z, p, \alpha, \beta}^{\wp, \kappa, \mu} \phi(z)\right| \\
& \leq \sum_{n=1}^{\infty}\left|\varphi_{n}\right| \frac{\Gamma(n+1)}{\Gamma(n+1-\wp)} \frac{\mathbf{B}_{p}^{\alpha, \beta, \kappa, \mu}(n+1,1-\wp)}{\mathbf{B}(n+1,1-\wp)}|z|^{n-\wp} \\
& \leq r^{-\wp} \sum_{n=1}^{\infty} \frac{\Gamma(n+1)}{\Gamma(n+1-\wp)} \frac{\mathbf{B}_{p}^{\alpha, \beta, \kappa, \mu}(n+1,1-\wp)}{\mathbf{B}(n+1,1-\wp)} r^{n} \\
& =r^{1-\wp} \sum_{n=0}^{\infty} \frac{\Gamma(n+2)}{\Gamma(n+2-\wp)} \frac{\mathbf{B}_{p}^{\alpha, \beta, \kappa, \mu}(n+2,1-\wp)}{\mathbf{B}(n+2,1-\wp)} r^{n} \\
& =\frac{r^{1-\wp}}{\Gamma(2-\wp)} \sum_{n=0}^{\infty} \frac{(1)_{n}(1)_{n}}{(2-\wp)_{n}} \frac{\mathbf{B}_{p}^{\alpha, \beta, \kappa, \mu}(n+2,1-\wp)}{\mathbf{B}(n+2,1-\wp)} \frac{r^{n}}{n !} \\
& =\frac{r^{1-\wp}}{\Gamma(2-\wp)} F_{p}^{\alpha, \beta, \kappa, \mu}(2,1 ; 2-\wp ; r ; 0) .
\end{aligned}
$$


This completes the proof.

Next, we proceed to find the upper bound of the operator (7). We have the following outcomes:

Theorem 2.3 Let $\phi \in \mathcal{S}$. Then

$$
\left.\left.\begin{array}{rl}
\left|D_{z, \rho, \alpha, \beta}^{\wp, \varsigma, \kappa, \mu} \phi(z)\right| \leq(\varsigma+1)^{\wp-1} r^{(1-\wp)} & \\
\cdot{ }_{1}^{\Psi_{1}}\left[\left(1+\frac{1}{(\varsigma+1)}, \frac{1}{(\varsigma+1)}\right) ;\right. \\
* F_{p}^{\alpha, \beta, \kappa, \mu}\left(2, \frac{\varsigma+2}{\varsigma+1} ; \frac{\varsigma+2}{\varsigma+1} ; r ; m-\wp\right)
\end{array}\right]\right)
$$

where the equality holds true for the Koebe function.

Proof. Consider the function $\phi(z) \in \mathcal{S}$ is read by (9). Thus, in view of Example 1.1, we conclude

$$
\begin{aligned}
& D_{z, p, \alpha, \beta}^{\wp, \varsigma, \kappa} \phi(z)=\sum_{n=1}^{\infty} \varphi_{n} D_{z, p, \alpha, \beta}^{\wp, \varsigma, \kappa, \mu} z^{n}, \varphi_{1}=1 \\
& =\sum_{n=1}^{\infty}\left[\begin{array}{c}
\varphi_{n} \frac{(\varsigma+1)^{\wp-1} \Gamma\left(\frac{n}{\varsigma+1}+1\right)}{\Gamma\left(\frac{n}{\varsigma+1}+1-\wp\right)} \\
\left.\times \frac{\mathbf{B}_{p}^{\alpha, \beta, \kappa, \mu}\left(\frac{n+\varsigma+1}{\varsigma+1}, 1-\wp\right)}{\mathbf{B}\left(\frac{n+\varsigma+1}{\varsigma+1}, 1-\wp\right)} z^{(1-\wp)(\varsigma+1)+n-1}\right]
\end{array}\right] .
\end{aligned}
$$

Thus by using the fact $\left|\varphi_{n}\right| \leq n$, we lead to

$$
\begin{aligned}
& \left|D_{z, p, \alpha, \beta}^{\wp, \varsigma, \kappa, \mu} \phi(z)\right| \\
& \leq \sum_{n=1}^{\infty}\left[\begin{array}{c}
\varphi_{n} \frac{(\varsigma+1)^{\wp-1} \Gamma\left(\frac{n}{\varsigma+1}+1\right)}{\Gamma\left(\frac{n}{\varsigma+1}+1-\wp\right)} \\
\left.\times \frac{\mathbf{B}_{p}^{\alpha, \beta, \kappa, \mu}\left(\frac{n+\varsigma+1}{\varsigma+1}, m-\wp\right)}{\mathbf{B}\left(\frac{n+\varsigma+1}{\varsigma+1}, m-\wp\right)}|z|^{(1-\wp)(\varsigma+1)+n-1}\right]
\end{array}\right] \\
& \leq r(1-\wp)(\varsigma+1)-1 \sum_{n=1}^{\infty}\left[\begin{array}{l}
n \frac{(\varsigma+1)^{\wp-1} \Gamma\left(\frac{n}{\varsigma+1}+1\right)}{\Gamma\left(\frac{n}{\varsigma+1}+1-\wp\right)} \\
\times \frac{\mathbf{B}_{p}^{\alpha, \beta, \kappa, \mu}\left(\frac{n+\varsigma+1}{\varsigma+1}, m-\wp\right)}{\mathbf{B}\left(\frac{n+\varsigma+1}{\varsigma+1}, m-\wp\right)} r^{n}
\end{array}\right]
\end{aligned}
$$

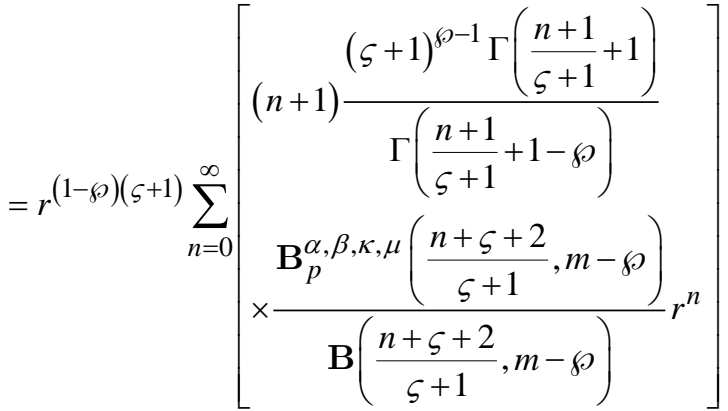

$$
\begin{aligned}
& =(\varsigma+1)^{\wp-1} r^{(1-\wp)(\varsigma+1)} \\
& \times \sum_{n=0}^{\infty}\left[\begin{array}{c}
\frac{\Gamma(n+2) \Gamma\left(\frac{n+1}{\varsigma+1}+1\right)}{\Gamma\left(\frac{n+1}{\varsigma+1}+1-\wp\right)} \\
\times \frac{\mathbf{B}_{p}^{\alpha, \beta, \kappa, \mu}\left(\frac{n+\varsigma+2}{\varsigma+1}, m-\wp\right)}{\mathbf{B}\left(\frac{n+\varsigma+2}{\varsigma+1}, m-\wp\right)} \frac{r^{n}}{n !}
\end{array}\right] \\
& =(\varsigma+1)^{\wp-1} r^{(1-\wp)}\left(\sum_{n=0}^{\infty} \frac{\Gamma\left(\frac{n+1}{\varsigma+1}+1\right)}{\Gamma\left(\frac{n+1}{\varsigma+1}+1-\wp\right)} \frac{r^{n}}{n !}\right) \\
& *\left(\sum_{n=0}^{\infty} \Gamma(n+2) \frac{\mathbf{B}_{p}^{\alpha, \beta, \kappa, \mu}\left(\frac{n+\varsigma+2}{\varsigma+1}, m-\wp\right)}{\mathbf{B}\left(\frac{n+\varsigma+2}{\varsigma+1}, m-\wp\right)} \frac{r^{n}}{n !}\right) \\
& =(\varsigma+1)^{\wp-1} r^{(1-\wp)}\left(\begin{array}{c}
{ }_{1} \Psi_{1}\left[\begin{array}{ll}
\left(1+\frac{1}{(\varsigma+1)}, \frac{1}{(\varsigma+1)}\right) ; \\
\left(1+\frac{1}{(\varsigma+1)}-\wp, \frac{1}{(\varsigma+1)}\right) ;
\end{array}\right] \\
* F_{p}^{\alpha, \beta, \kappa, \mu}(2, b ; b ; r ; m-\wp)
\end{array}\right] .
\end{aligned}
$$

This completes the proof.

Theorem 2.4 Let $\phi(z) \in \mathcal{K}$. Then

$$
\begin{aligned}
& \left|D_{z, p, \alpha, \beta}^{\wp, \varsigma, \kappa, \mu} \phi(z)\right| \\
& \leq(\varsigma+1)^{\varsigma-1} r^{(1-\wp)} \\
& \times\left(\begin{array}{cc}
{ }_{1} \Psi_{1}\left[\begin{array}{ll}
\left(1+\frac{1}{(\varsigma+1)}, \frac{1}{(\varsigma+1)}\right) ; & r \\
\left(1+\frac{1}{(\varsigma+1)}-\wp, \frac{1}{(\varsigma+1)}\right) ; &
\end{array}\right] \\
* F_{p}^{\alpha, \beta, \kappa, \mu}(1, b ; b ; r ; 1-\wp)
\end{array}\right) \\
& (r=|z| ; \quad z \in U ; \quad 0<\wp<1) \text {, }
\end{aligned}
$$

where the equality holds true for the Koebe function.

Proof. Suppose that the function $\phi(z) \in \mathcal{K}$, i.e. $\left(\left|\varphi_{n}\right| \leq 1\right)$ is written by (9). Then, we conclude 
$\left|D_{z, p, \alpha, \beta}^{\wp, \varsigma, \mu} \phi(z)\right|$

$\leq \sum_{n=1}^{\infty}\left[\begin{array}{c}\left|\varphi_{n}\right| \frac{(\varsigma+1)^{\wp-1} \Gamma\left(\frac{n}{\varsigma+1}+1\right)}{\Gamma\left(\frac{n}{\varsigma+1}+1-\wp\right)} \\ \times \frac{\mathbf{B}_{p}^{\alpha, \beta, \kappa, \mu}\left(\frac{n+\varsigma+1}{\varsigma+1}, 1-\wp\right)}{\mathbf{B}\left(\frac{n+\varsigma+1}{\varsigma+1}, 1-\wp\right)}|z|^{(1-\wp)(\varsigma+1)+n-1}\end{array}\right]$

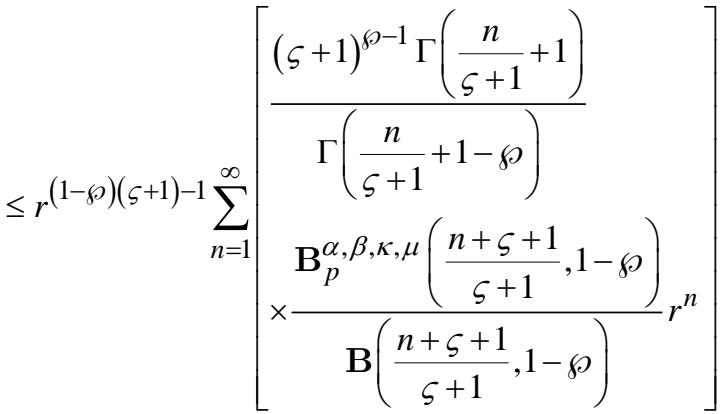

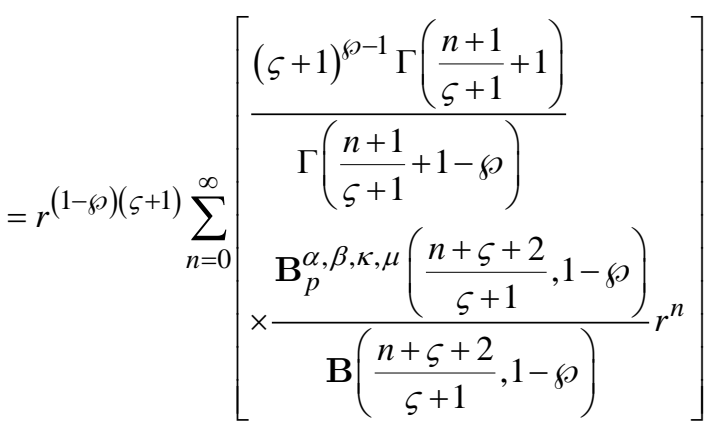

$=(\varsigma+1)^{\wp-1} r^{(1-\wp)(\varsigma+1)} \sum_{n=0}^{\infty}\left[\begin{array}{l}\frac{\Gamma(n+1) \Gamma\left(\frac{n+1}{\varsigma+1}+1\right)}{\varsigma\left(\frac{n+1}{\varsigma+1}+1-\wp\right)} \\ \times \frac{\mathbf{B}_{p}^{\alpha, \beta, \kappa, \mu}\left(\frac{n+\varsigma+2}{\varsigma+1}, 1-\wp\right)}{\mathbf{B}\left(\frac{n+\varsigma+2}{\varsigma+1}, 1-\wp\right)} \frac{r^{n}}{n !}\end{array}\right]$

$=(\varsigma+1)^{\wp-1} r^{(1-\wp)}\left(\sum_{n=0}^{\infty} \frac{\Gamma\left(\frac{n+1}{\varsigma+1}+1\right)}{\Gamma\left(\frac{n+1}{\varsigma+1}+1-\wp\right)} \frac{r^{n}}{n !}\right)$

$*\left(\sum_{n=0}^{\infty} \Gamma(n+1) \frac{\mathbf{B}_{p}^{\alpha, \beta, \kappa, \mu}\left(\frac{n+\varsigma+2}{\varsigma+1}, 1-\wp\right)}{\mathbf{B}\left(\frac{n+\varsigma+2}{\varsigma+1}, 1-\wp\right)} \frac{r^{n}}{n !}\right)$

$=(\varsigma+1)^{\wp-1} r^{(1-\wp)}\left(\begin{array}{l}{ }_{1} \Psi_{1}\left[\begin{array}{ll}\left(1+\frac{1}{(\varsigma+1)}, \frac{1}{(\varsigma+1)}\right) ; \\ \left(1+\frac{1}{(\varsigma+1)}-\wp, \frac{1}{(\varsigma+1)}\right) ;\end{array}\right] \\ * F_{p}^{\alpha, \beta, \kappa, \mu}(1, b ; b ; r ; 1-\wp)\end{array}\right]$.

This completes the proof.
Various significant functions in different areas (which are commonly recognized as special functions) are formulated through infinite series or improper integrals (or infinite products such as convolution product). Over previous four decades or so, numerous motivating and beneficial generalizations of many of the acquainted special functions (such as the Gauss hypergeometric function, the Gamma and Beta functions, etc.) have been reflected by several studies not only in mathematics, but also in physics, computer sciences and engineering (see $[8,9])$. The aforesaid works have widely fortified our present study.

\subsection{Differential Operators}

The fractional integral and derivative operators, including different types and classes of special functions have faced applications and importance in many fields and subjects, such as mathematical analysis, dynamic systems, statistics, computer sciences and mathematical physics. Recently, numerous researchers have improved, generalized and modified different fractional derivative forms of the Riemann-Liouville operators. In this section, we introduce two generalized fractional differential operator in the sense of Tremblay operator by utilizing the general forms that described in the above section.

In [10], Tremblay formulated and investigated a fractional calculus operator defined in term of the Riemann-Liouville fractional differential operator. This operator was extending into the complex domain to include $D_{Z}^{\wp}$ as follows [11]:

$$
T_{Z}^{\wp, \gamma}:=\frac{\Gamma(\gamma+1)}{\Gamma(\wp+1)} z^{1-\gamma} D_{Z}^{\wp-\gamma} z^{\wp-1},
$$

where

$$
(\wp, \gamma \in[0,1] ; \wp>\gamma ; z \in \mathbb{C}) .
$$

Now based on the generalized operators (7) and (9), we have the following new operators:

$$
\begin{aligned}
T_{z, p, \alpha, \beta}^{\wp, \gamma, \kappa, \mu}:= & \frac{\Gamma(\gamma+1)}{\Gamma(\wp+1)} \frac{\mathbf{B}(\wp+1,1-\wp+\gamma)}{\mathbf{B}_{p}^{\alpha, \beta, \kappa, \mu}(\wp+1,1-\wp+\gamma)} \\
& \times z^{1-\gamma} D_{z, p, \alpha, \beta}^{\wp-\gamma, \kappa, \mu} z^{\wp-1},
\end{aligned}
$$

and

$$
T_{Z, p, \alpha, \beta}^{\wp, \gamma, \varsigma, \kappa, \mu}:=\Theta^{\wp, \gamma, \varsigma, \kappa, \mu} z^{1-\gamma-(1-\wp+\gamma) \varsigma} D_{z, p, \alpha, \beta}^{\wp-\gamma, \varsigma, \kappa, \mu} z^{\wp-1},
$$

where

$$
\begin{aligned}
\Theta^{\wp, \gamma, \varsigma, \kappa, \mu}:= & \frac{\Gamma\left(\gamma+1-\wp+\frac{\wp}{\varsigma+1}\right)}{(\varsigma+1)^{\wp-1-\gamma} \Gamma\left(\frac{\wp}{\varsigma+1}+1\right)} \\
& \times \frac{\mathbf{B}\left(\frac{\wp+\varsigma+1}{\varsigma+1}, 1-\wp+\gamma\right)}{\mathbf{B}_{p}^{\alpha, \beta, \kappa, \mu}\left(\frac{\wp+\varsigma+1}{\varsigma+1}, 1-\wp+\gamma\right)} .
\end{aligned}
$$

Note that by the boundedness of the operators (7) and (9) for some special geometric functions, the last two 
operators are bounded in the unit open disk. Moreover, we have the following properties:

Theorem $2.5 T_{z, p, \alpha, \beta}^{\wp, \gamma, \kappa, \mu}: \mathcal{A} \rightarrow \mathcal{A}$.

Proof. Let $\phi \in \mathcal{A}$ then, in view of Example 1.1, we obtain

$$
\begin{aligned}
& T_{z, p, \alpha, \beta}^{\wp, \gamma, \kappa, \mu} \phi(z) \\
& =\frac{\Gamma(\gamma+1)}{\Gamma(\wp+1)} \frac{\mathbf{B}(\wp+1,1-\wp+\gamma)}{\mathbf{B}_{p}^{\alpha, \beta, \kappa, \mu}(\wp+1,1-\wp+\gamma)} \\
& \times z^{1-\gamma} D_{z, p, \alpha, \beta}^{\wp-\gamma, \kappa, \mu} z^{\wp-1} \phi(z) \\
& =\frac{\Gamma(\gamma+1)}{\Gamma(\wp+1)} \frac{\mathbf{B}(\wp+1,1-\wp+\gamma)}{\mathbf{B}_{p}^{\alpha, \beta, \kappa, \mu}(\wp+1,1-\wp+\gamma)} z^{1-\gamma} \\
& \times\left[D_{z, p, \alpha, \beta}^{\wp-\gamma, \kappa, \mu} z^{\wp}+\sum_{n=2}^{\infty} \varphi_{n} D_{z, p, \alpha, \beta}^{\wp-\gamma, \kappa, \mu} z^{\wp-1+n}\right] \\
& =\frac{\Gamma(\gamma+1)}{\Gamma(\wp+1)} \frac{\mathbf{B}(\wp+1,1-\wp+\gamma)}{\mathbf{B}_{p}^{\alpha, \beta, \kappa, \mu}(\wp+1,1-\wp+\gamma)} z^{1-\gamma} \\
& \times\left[\begin{array}{l}
\frac{\Gamma(\gamma+1)}{\Gamma(\wp+1)} \frac{\mathbf{B}(\wp+1,1-\wp+\gamma)}{\mathbf{B}_{p}^{\alpha, \beta, \kappa, \mu}(\wp+1,1-\wp+\gamma)} z^{\gamma} \\
+\sum_{n=2}^{\infty} \varphi_{n} D_{z, p, \alpha, \beta}^{\wp-\gamma, \kappa, \mu} z^{\wp-1+n}
\end{array}\right] \\
& =z+\sum_{n=2}^{\infty} \Phi_{n} z^{n},
\end{aligned}
$$

where

$$
\begin{aligned}
\Phi_{n}:= & \varphi_{n} \frac{\Gamma(\gamma+1)}{\Gamma(\wp+1)} \frac{\mathbf{B}(\wp+1,1-\wp+\gamma)}{\mathbf{B}_{p}^{\alpha, \beta, \kappa, \mu}(\wp+1,1-\wp+\gamma)} \\
& \times \frac{\Gamma(\wp+n)}{\Gamma(n+\gamma)} \frac{\mathbf{B}_{p}^{\alpha, \beta, \kappa, \mu}(\wp+n, 1-\wp+\gamma)}{\mathbf{B}(\wp+n, 1-\wp+\gamma)}, \quad n \geq 2 .
\end{aligned}
$$

Hence $T_{z, p, \alpha, \beta}^{\wp, \gamma, \kappa, \mu} \in \mathcal{A}$.

Corollary 2.1 Let $\phi(z) \in \mathcal{A}$. If

$$
\varphi_{n}:=\frac{\left[\begin{array}{l}
n \Gamma(\wp+1) \mathbf{B}_{p}^{\alpha, \beta, \kappa, \mu}(\wp+1,1-\wp+\gamma) \\
\times \Gamma(n+\gamma) \mathbf{B}(\wp+n, 1-\wp+\gamma)
\end{array}\right]}{\left[\begin{array}{l}
\Gamma(\gamma+1) \mathbf{B}(\wp+1,1-\wp+\gamma) \\
\times \mathbf{B}_{p}^{\alpha, \beta, \kappa, \mu}(\wp+n, 1-\wp+\gamma) \Gamma(\wp+n)
\end{array}\right]}, n \geq 2
$$

then $T_{z, p, \alpha, \beta}^{\wp, \gamma, \kappa, \mu}: \mathcal{A} \rightarrow \mathcal{S}$.

Corollary 2.2 Let $\phi(z) \in \mathcal{A}$ satisfying the condition of Corollary 2.1. If $\wp=\gamma, p=0$ then $\phi(z) \in \mathcal{S}$.

Corollary 2.3 Let $\phi(z) \in \mathcal{A}$. If

$$
\varphi_{n}:=\frac{\left[\begin{array}{l}
\Gamma(\wp+1) \mathbf{B}_{p}^{\alpha, \beta, \kappa, \mu}(\wp+1,1-\wp+\gamma) \\
\times \Gamma(n+\gamma) \mathbf{B}(\wp+n, 1-\wp+\gamma)
\end{array}\right]}{\left[\begin{array}{l}
\Gamma(\gamma+1) \mathbf{B}(\wp+1,1-\wp+\gamma) \\
\times \mathbf{B}_{p}^{\alpha, \beta, \kappa, \mu}(\wp+n, 1-\wp+\gamma) \Gamma(\wp+n)
\end{array}\right]}, n \geq 2
$$

then $T_{z, p, \alpha, \beta}^{\wp, \gamma, \kappa, \mu}: \mathcal{A} \rightarrow \mathcal{K}$.

Corollary 2.4 Let $\phi(z) \in \mathcal{A}$ satisfying the condition of Corollary 2.3. If $\wp=\gamma, p=0$ then $\phi(z) \in \mathcal{K}$.

Theorem $2.6 T_{z, p, \alpha, \beta}^{\wp, \gamma, \varsigma, \kappa}: \mathcal{A} \rightarrow \mathcal{A}$.

Proof. Let $\phi \in \mathcal{A}$ then, in view of Example 1.2, we have

$T_{z, p, \alpha, \beta}^{\wp, \gamma, \kappa, \mu} \phi(z)$

$=\Theta^{\wp, \gamma, \varsigma, \kappa, \mu} z^{1-\gamma-(1-\wp+\gamma) \varsigma}$

$\times\left[D_{z, p, \alpha, \beta}^{\wp-\gamma, \varsigma, \kappa, \mu} z^{\wp}+\sum_{n=2}^{\infty} \varphi_{n} D_{z, p, \alpha, \beta}^{\wp-\gamma, \varsigma, \kappa, \mu} z^{\wp-1+n}\right]$

$=\Theta^{\wp, \gamma, \varsigma, \kappa, \mu} Z^{1-\gamma-(1-\wp+\gamma) \varsigma}$

$\times\left[\frac{1}{\Theta^{\wp, \gamma, \varsigma, \kappa, \mu}} z^{\gamma+(1-\wp+\gamma) \varsigma}+\sum_{n=2}^{\infty} \varphi_{n} D_{z, p, \alpha, \beta}^{\wp-\gamma, \varsigma, \kappa, \mu} z^{\wp-1+n}\right]$

$=z+\sum_{n=2}^{\infty} \Psi_{n} z^{n}$

where

$$
\begin{aligned}
\Psi_{n}:= & \Theta^{\wp, \gamma, \varsigma, \kappa, \mu} \varphi_{n} \frac{(\wp+1)^{\wp-\gamma-1} \Gamma\left(\frac{\wp-1+n}{\varsigma+1}+1\right)}{\Gamma\left(\frac{\wp-1+n}{\varsigma+1}+1-\wp+\gamma\right)} \\
& \times \frac{\mathbf{B}_{p}^{\alpha, \beta, \kappa, \mu}\left(\frac{\wp-1+n+\varsigma+1}{\varsigma}, 1-\wp+\gamma\right)}{\mathbf{B}\left(\frac{\wp-1+n+\varsigma+1}{\varsigma}, 1-\wp+\gamma\right)}, n \geq 2 .
\end{aligned}
$$

Corollary 2.5 Let $\phi(z) \in \mathcal{A}$. If

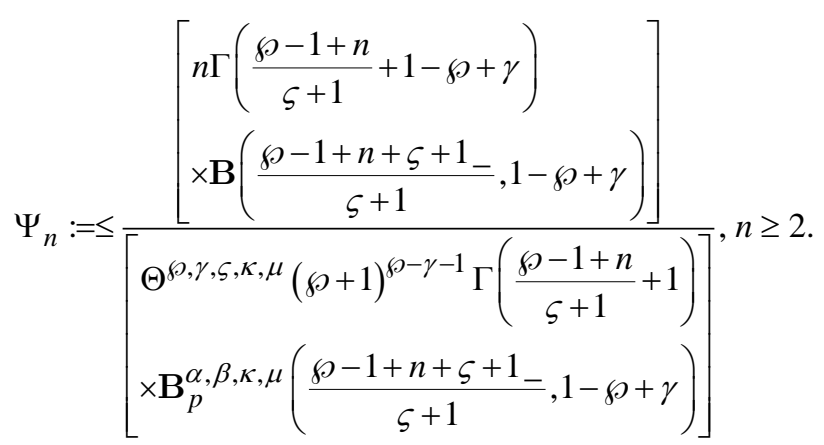

then $T_{z, p, \alpha, \beta}^{\wp, \gamma, \kappa, \mu}: \mathcal{A} \rightarrow \mathcal{S}$.

Corollary 2.6 Let $\phi(z) \in \mathcal{A}$ satisfying the condition of Corollary 2.5. If $\wp=\gamma, p=0$ then $\phi(z) \in \mathcal{S}$.

Corollary 2.7 Let $\phi(z) \in \mathcal{A}$. If

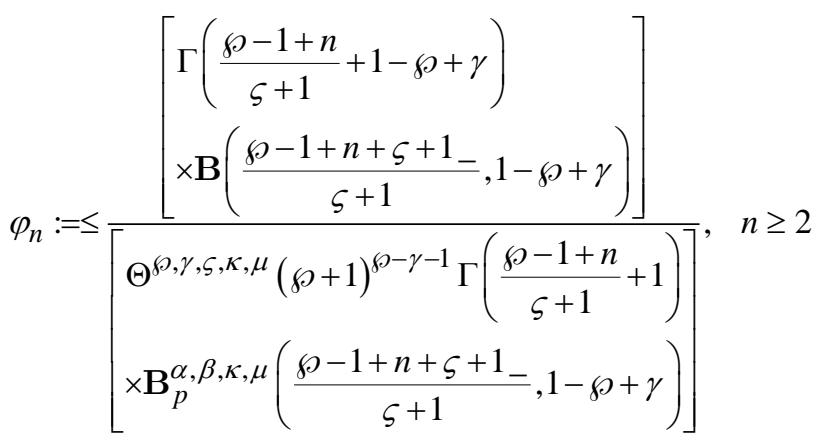


then $T_{z, p, \alpha, \beta}^{\wp, \gamma, \kappa, \mu}: \mathcal{A} \rightarrow \mathcal{K}$.

Corollary 2.8 Let $\phi(z) \in \mathcal{A}$, satisfying the condition of Corollary 2.7. If $\wp=\gamma, p=0$ then $\phi(z) \in \mathcal{K}$.

\section{Conclusion}

From above, we imposed a generalization of one of the most important fractional operators (Srivastava-Owa fractional differential operator and its alternative) in an arbitrary order in the unit disk. We conclude that the generalize differential operator satisfying the distortion property under some special classes of analytic functions. Motivated by the numerous allowances of the fractional derivative operators which have newly been reflected by many authors, here, we purposed two operators involving the generalized hypergeometric-type function and the generalized Beta function. Various studies can be found in $[12,13,14]$.

\section{Conflict of Interests}

The authors declare that there is no conflict of interests regarding the publication of this article.

\section{Authors Contribution}

Both authors jointly worked on deriving the results and approved the final manuscript.

\section{Acknowledgment}

The authors would like to thank the referees for giving useful suggestions for improving the work. This research is supported by Project No.: RG312-14AFR from the University of Malaya.

\section{References}

[1] Ibrahim RW, Sokol J: On a new class of analytic function derived by a fractional differential operator Acta Mathematica Scientia 2014, 34B(4):110.

[2] Ibrahim RW, Sokol J: A geometric property for a class of meromorphic analytic functions. Journal of Inequalities and Applications 2014, 2014:120.

[3] Srivastava HM, Owa S: Univalent Functions, Fractional Calculus, and Their Applications, Halsted Press, John Wiley and Sons, New York, Chichester, Brisban, and Toronto, 1989.

[4] Ibrahim RW, On generalized Srivastava-Owa fractional operators in the unit disk, Advances in Difference Equations 2011, 2011:55.

[5] Srivastava HM, Agarwal P, Jain S, Generating functions for the generalized Gauss hypergeometric functions, Appl. Math. Comput. 247, 2014: 348-352.

[6] Agarwal P, Choi J, Paris RB, Extended Riemann-Liouville fractional derivative operator and its applications, J. Nonlinear Sci. Appl. 8 2015: 451-466.

[7] Srivastava HM, Karlsson PW: Multiple Gaussian Hypergeometric Series, Halsted Press (Ellis Horwood Limited, Chichester), John Wiley and Sons, New York, Chichester, Brisbane and Toronto, 1985.

[8] Özergin E, Özarslan MA, Altin A: Extension of gamma, beta and hypergeometric functions, J. Comput. Appl. Math. 235, 2011: 4601-4610.

[9] Ibrahim RW, Studies on Generalized Fractional Operators in Complex Domain, Mathematics Without Boundaries Lecture notice Book ID: 272027, Springer, 2014: 1-15.

[10] Tremblay R, Une contribution a la theori de la derivee fractionnaire [Ph.D. thesis ]. Quebec. Canada: Laval University; 1974.

[11] Ibrahim RW, Jahangiri M, Boundary fractional differential equation in a complex domain, Boundary Value Problems 2014, 2014:66.

[12] Ibrahim RW, et al., Third-order differential subordination and superordination involving a fractional operator, Open Math. 13, 2015: 706-728.

[13] Ibrahim RW, et al., Upper and lower bounds of integral operator defined by the fractional hypergeometric function, Open Math. 13, 2015: 768-780.

[14] Magesh N., et al., Subordination and superordination results associated with the generalized hypergeometric function, Math. Slovaca, 64, 2014: 1197-1216. 